\title{
Terahertz-Magnetic-Field Induced Ultrafast Faraday Rotation of Molecular Liquids
}

\author{
Vasileios Balos@, Genaro Bierhance, Martin Wolf, and Mohsen Sajadi ${ }^{*}$ \\ Fritz Haber Institute of the Max Planck Society, Faradayweg 4-6, 14195 Berlin, Germany
}

(Received 10 October 2019; accepted 29 January 2020; published 6 March 2020)

\begin{abstract}
Rotation of the plane of the polarization of light in the presence of a magnetic field, known as the Faraday rotation, is a consequence of the electromagnetic nature of light and has been utilized in many optical devices. Current efforts aim to realize the ultrafast Faraday rotation on a subpicosecond timescale. Thereby, the Faraday medium should allow an ultrafast process by which in the presence of an ultrashort intense magnetic field, the light polarization rotates. We meet these criteria by applying an intense single cycle $\mathrm{THz}$ magnetic field to simple molecular liquids and demonstrate the rotation of the plane of polarization of an optical pulse traversing the liquids on a subpicosecond timescale. The effect is attributed to the deflection of an optically induced instantaneous electric polarization under the influence the $\mathrm{THz}$ magnetic field. The resolved Faraday rotation scales linearly with the THz magnetic field and quadratically with the molecular polarizability.
\end{abstract}

DOI: 10.1103/PhysRevLett.124.093201

When a linearly polarized electromagnetic (EM) wave, along with a magnetic field, propagates through an optically transparent medium its plane of polarization rotates. This effect is termed Faraday rotation and has played an important role in elucidating the EM nature of light [1]. It has also been extensively used in many devices including optical switches [2], optical communication systems [3], quantum memories [4], nuclear magnetic resonance spectrometers [5], and light modulators [6]. Further applications of Faraday rotators rely on the development in two main areas, introducing new Faraday media or exploring new features for existing materials and increasing the modulation speed of Faraday rotators.

So far there has been great progress in finding new materials with large Faraday rotation, such as nitride semiconductor alloys [7,8], two-dimensional electron gases [9], graphene [10], and organic molecules [11]. However, a subpicosecond light modulation requires an inherently ultrafast microscopic process whereupon the light polarization rotates [12]. For example, in semiconductors the modulation rate is limited by the mobility of the charge carriers and the relaxation processes in the bulk material [13]. Moreover, ultrafast magnetic switching with a terahertz $(\mathrm{THz})$ clock rate is required, a goal that is far to reach with current magnetic pulses with temporal duration of about hundreds of picoseconds $[14,15]$.

Published by the American Physical Society under the terms of the Creative Commons Attribution 4.0 International license. Further distribution of this work must maintain attribution to the author(s) and the published article's title, journal citation, and DOI. Open access publication funded by the Max Planck Society.
To increase the modulation speed of the Faraday rotators, we take advantage of the magnetic field of single cycle $\mathrm{THz}$ EM pulses. The potential of $\mathrm{THz}$ pulses has so far been explored to observe [16] and in some cases control the lowenergy elementary excitations in liquids [17,18], solids [19], and gases [20] on ultrafast timescale.

In these examples, the $\mathrm{THz}$ electric field interacts with the IR-active dipolar excitations of the samples, while the THz-magnetic-field interaction is neglected because of its much weaker interaction energy. However, recent studies have shown the great potential of the THz magnetic fields for ultrafast Faraday rotation via their direct coupling to the magnons in antiferromagnetic and paramagnetic materials $[21,22]$. Here, we aim to diversify the realm of ultrafast Faraday rotators beyond the spin interactions and demonstrate the THz-magnetic-field induced ultrafast Faraday rotation in molecular liquids at ambient conditions. We show that in liquids, an instantaneous polarization induced by a femtosecond optical pulse acts as an ultrashort current burst, which in the presence of a $\mathrm{THz}$ magnetic field, rotates the polarization of the optical pulse. This process in highly polarizable liquids, such as long chain alcohols and alkanes, is more efficient than that of the standard magneto-optic media with giant Verdet constant such as terbium gallium garnet (TGG) [23].

Our experimental setup follows the geometry, introduced by Hoffmann et al. in their pioneering $\mathrm{THz}$ Kerr effect work [17]. As shown in Fig. 1(a), two EM waves with central frequencies $\sim 1 \mathrm{THz}(300 \mu \mathrm{m})$ and $\sim 374 \mathrm{THz}$ $(0.8 \mu \mathrm{m})$ and variable time delay are incident on the samples with a wide angle of $\gamma \approx 40^{\circ}$. To avoid contamination of the measured signals by a window response of a cuvette, we hold the liquids in an open-top bucket whose 


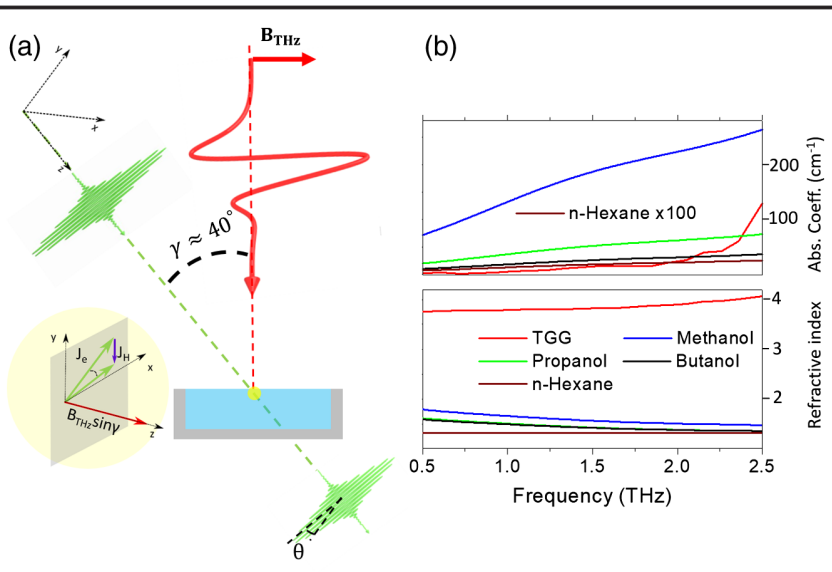

FIG. 1. (a) A femtosecond optical pulse is mixed under a wide angle of $\sim 40^{\circ}$ with a single cycle linearly polarized $\mathrm{THz}$ pulse on liquids. The optically induced instantaneous polarization acts as a current burst $\mathbf{J}_{e}(t)$. This current is bent by the $\mathrm{THz}$ magnetic field in the plane perpendicular to the optical pulse propagation direction. The deflected component of the current, i.e., $\mathbf{J}_{H}(t)$ causes the rotation of the optical pulse polarization. The liquids are held in an open-top bucket. In this configuration, the $\mathrm{THz}$ pulse impinges the sample vertically. (b) $\mathrm{THz}$ absorption and refractive index of some of the samples used in this study. For the TGG crystal, data is obtained from our $\mathrm{THz}$ time-domain spectrometer. The refractive index and absorption coefficient of methanol [24], butanol, propanol [25], and $n$-hexane [26] are calculated from their dielectric permittivity and loss spectra of the cited references.

bottom side is transparent at $800 \mathrm{~nm}$ and the $\mathrm{THz}$ pulse propagates vertically towards the samples.

The optical probe pulse has $\sim 2 \mathrm{~nJ}$ energy and $\sim 10$ fs temporal duration. The $\mathrm{THz}$ pulse is generated by the rectification of a $800 \mathrm{~nm}$ optical pulse $(60 \mathrm{fs}, 5 \mathrm{~mJ}$, $1 \mathrm{kHz}$ repetition rate) in a $\mathrm{LiNbO}_{3}$ crystal using the tilted-pulse-front technique [27,28]. The single-cycle $\mathrm{THz}$ pulse is phase locked, linearly polarized, and when focused to a diameter of $\sim 0.6 \mathrm{~mm}$ produces an electric-field $E(t) \approx$ $2 \mathrm{MV} / \mathrm{cm}$ corresponding to a magnetic field of $B(t)=$ $E(t) / c \approx 0.7 \mathrm{~T}$, where $c$ is the speed of light.

The resulting transient effect is obtained by measuring the polarization change of the optical pulse using a set of balanced photodiodes. Because of the THz pump-induced birefringence, the linear polarization of the traversing optical probe pulse with wavelength $\lambda$, through a sample with thickness $L$ acquires rotation and ellipticity. The rotation (ellipticity) of polarization $\Delta \theta(\Delta \phi)$ is proportional to the phase difference of the left- vs right-handed circularly (parallel vs perpendicular linearly) polarized components of the probe pulse $[29,30]$

$$
\begin{aligned}
& \Delta \theta=\frac{\lambda}{2 \pi L}\left(n_{L}-n_{R}\right), \\
& \Delta \varphi=\frac{\lambda}{2 \pi L}\left(n_{\|}-n_{\perp}\right),
\end{aligned}
$$

(a)

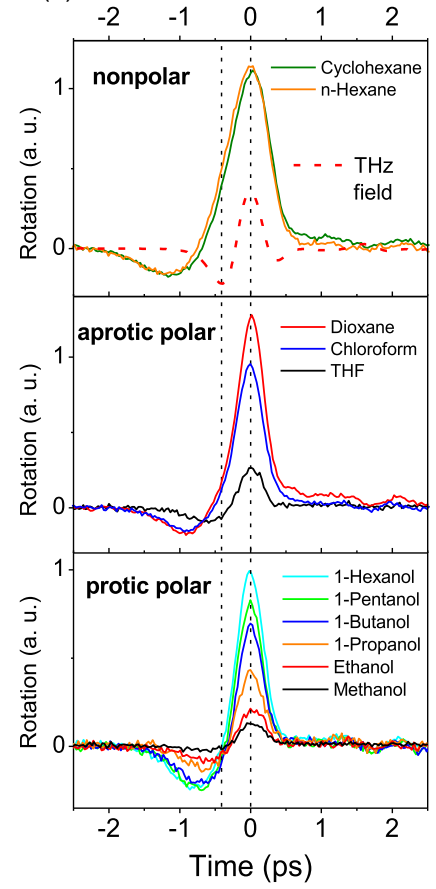

(b)

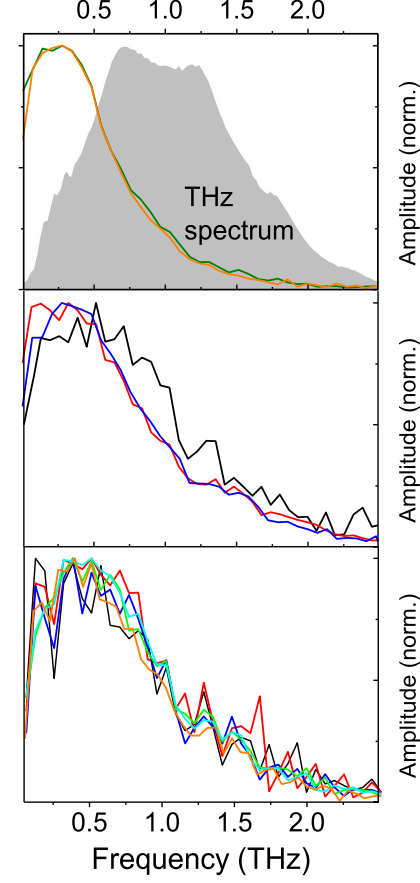

FIG. 2. (a) Faraday rotation of a series of molecular liquids at room temperature. Nonpolar, cyclohexane (dark green) and $n$ hexane (red); aprotic polar, dioxane (pink), chloroform (blue), tetrahydrofuran (THF, black); protic polar, 1-hexanol (cyan), 1-pentanol (green), 1- butanol (blue), 1-propanol (pink), ethanol (red) and methanol (black). The measured signals resemble the $\mathrm{THz}$ waveform (dashed line). (b) The Fourier spectra of the signals in panel (a). The gray area shows the amplitude spectrum of the THz pulse.

where $n_{L}$ and $n_{R}\left(n_{\|}\right.$and $\left.n_{\perp}\right)$ are, respectively, the refractive indices of the material for the circularly polarized left- and right-handed (linearly polarized parallel and perpendicular) components of the optical probe pulse. The $\Delta \theta(\Delta \phi)$ is detected with a combination of a halfwave plate (quarter-wave plate) and a Wollaston prism that splits the incoming beam into two perpendicularly polarized beams with power $P_{\|}$and $P_{\perp}$. In the limit $|\Delta \theta|$ and $|\Delta \phi| \ll 1$, the normalized difference $P_{\|}-P_{\perp} \propto \Delta \theta(\Delta \phi)$ is measured by two photodiodes as a function of the time delay between the $\mathrm{THz}$ and the optical pulses [26,31]. The utilized liquids, are all spectroscopic grade with purity $>99 \%$. The absorption coefficient and refractive index of some of the polar and nonpolar liquids and also the TGG crystal (0.5 mm thick) are given in Fig. 1(b).

Figure 2(a) shows the measured Faraday rotation $\Delta \theta$ of the optical probe pulse for all liquids. For comparison, the $\mathrm{THz}$ pulse is given by a dashed red line. All signals are bipolar and to a large extent resemble the THz waveform. The Fourier spectrum of the signals is given in Fig. 2(b). The gray area shows the spectrum of the $\mathrm{THz}$ pulse. The results clearly show, the $\mathrm{THz}$ induced rotation of the probe polarization in the subpicosecond timescale. Note also that 

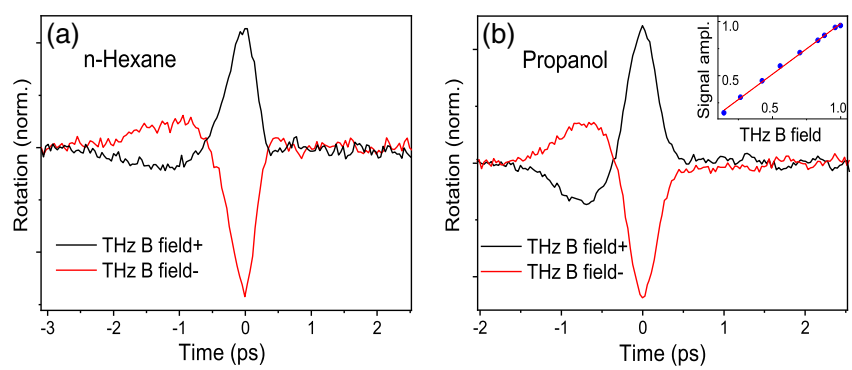

FIG. 3. Faraday rotation of $n$-hexane (a) and propanol (b) scales linearly with the applied $\mathrm{THz}$ magnetic field. In both samples the measured signal flips sign, by $180^{\circ}$ phase shift of the $\mathrm{THz}$ magnetic field. Inset in panel (b) shows the fluence dependence of the peak-to-peak amplitude of the rotation signal of propanol.

the observed rotation signal is independent of the probe polarization. As shown in Fig. S2 in the Supplemental Material [32], identical signals are obtained for $P$ and $S$ polarized optical probe pulses. We further determine the dependence of $\Delta \theta$ on the THz field, by driving the liquids with $180^{\circ}$ phase-shifted THz pulses. As shown in Fig. 3, $\Delta \theta$ signals of $n$-hexane and propanol flip sign when flipping the $\mathrm{THz}$ field polarity, indicating that $\Delta \theta$ scales linearly with the $\mathrm{THz}$ field. The fluence dependence result, shown in the inset of Fig. 3(b), corroborates the latter finding, too.

Interestingly, for water both the ellipticity [33] and the rotation signals are bipolar. However, as shown in Fig. S1 in the Supplemental Material [32], while the ellipticity signal remains intact, the rotation signal flips sign by shifting the phase of the $\mathrm{THz}$ pulse by $180^{\circ}$, declaring the different nature of the two effects. The unipolar $\mathrm{THz}$ electric-field-induced ellipticity of the rest of the liquids has been published elsewhere [18,34].

Additionally, we compared the polarization rotation of liquids with that of a standard Faraday medium with very high magneto-optical Verdet constant [22]. As shown in Fig. 4, the TGG Faraday rotation has a bipolar shape [35] but, because of a large velocity mismatch between $\mathrm{THz}$ and optical pulses, the measured response is broadened. This broadening can be accurately simulated by $\Delta \theta=(2 \pi / \lambda) \int_{0}^{L} d x \Delta n(0, t+\beta x)$, where the inversevelocity mismatch $\beta=v_{\mathrm{opt}}^{-1}-v_{\mathrm{THz}}^{-1}$ quantifies the temporal walk-off of the pump and probe pulses per propagation length [36].

To elucidate the nature of the light-matter interaction causing the rotation signal of liquids, we refer to the electric Hall effect by which the deflection of an electric current in the presence of an external magnetic field is explained. In a simple classical picture, the Lorentz force $e \mathbf{v} \times \mathbf{B}_{0}$ bends the electric current out of the plane of a static magnetic field $\mathbf{B}_{0}$ and the velocity vector $\mathbf{v}$ of the electric charge $e$. Interestingly, in its optical analog, i.e., the optical Hall effect [37], the bending of the dielectric displacement—due to the Lorentz force-is monitored by the rotation of the

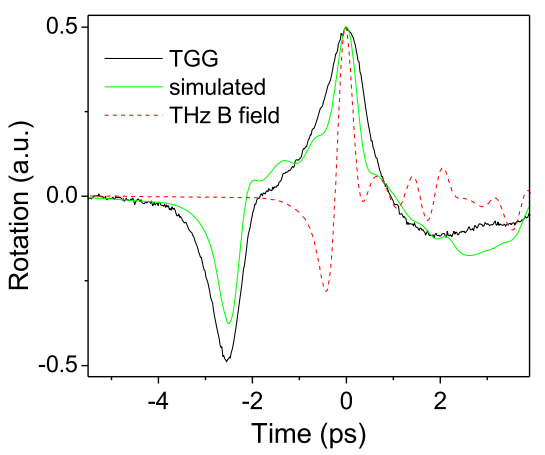

FIG. 4. The magneto-optic Faraday rotation of a terbium gallium garnet crystal and the simulated signal in which the velocity mismatch between the $\mathrm{THz}$ and optical pulses is taken into account (see text). The dashed line shows the THz magnetic field.

light polarization passing through or reflected from the dielectric medium.

In our molecular counterpart of the electric and the optical Hall effects, the $\mathrm{THz}$ magnetic field deflects an optically induced instantaneous electric current in molecules, resulting in an optical polarization rotation. In the optical Hall configuration, the directions of the magnetic field and the optical wave vector are parallel, whereas in our experiment we deviate from this scheme by an angle of $\sim 40^{\circ}$ between the two EM pulses to attain high time resolution. As a result, the dynamics up to $\sim 2 \mathrm{THz}$ can be resolved; see Fig. 2(b). Accordingly, the Lorentz force is proportional to the projected component of the $\mathrm{THz}$ magnetic field into the direction of the optical pulse propagation; see Fig. 1(a).

Upon propagation of an optical pulse in a dielectric medium, an instantaneous polarization $\mathbf{P}_{e}(t)=\alpha \mathbf{E}_{\text {opt }}(t)$ is induced which scales with the polarizability $\alpha$ and electric field $\mathbf{E}_{\text {opt }}(t)$ of the optical pulse. For simplicity, we assume that the molecules are linear rotors with their largest polarizability tensor element along the symmetry axis of molecules, which is fairly accurate for the long chain alcohols and $n$-alkanes [38]. The optical-field-induced polarization can be seen as a molecular current [39], given by the time derivative of $\mathbf{P}_{e}(t)$,

$$
\mathbf{J}_{e}(t)=\partial_{t} \mathbf{P}_{e}(t)
$$

A component of the THz magnetic field $\mathbf{B}_{\mathrm{THz}}(t)$, parallel to the propagation direction of the optical pulse, exerts a Lorentz force to the electric current and bends the current $\mathbf{J}_{e}(t)$ in the plane perpendicular to the optical pulse wave vector. The deflected component of the electric current, namely, the Hall current, reads

$\mathbf{J}_{H}(t)=\mathbf{J}_{e}(t) \times \mathbf{B}_{\mathrm{THz}}(t) \sin \gamma=\partial_{t} \mathbf{P}_{e}(t) \times \mathbf{B}_{\mathrm{THz}}(t) \sin \gamma$. 


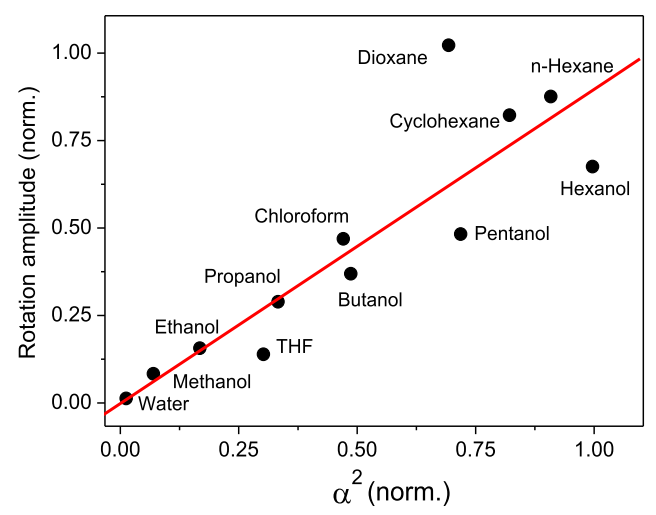

FIG. 5. Peak-to-peak amplitude of the Faraday rotation signal of liquids is plotted vs the square of the average molecular polarizability of liquids, taken from the following references: water [42], cyclohexane [43], chloroform, methanol, ethanol, propanol, butanol, pentanol, hexanol, $n$-hexane, dioxane [38], and tetrahydrofuran (THF) [44].

Now the bended current, $\mathbf{J}_{e}(t)+\mathbf{J}_{H}(t)$ is the source of the electromagnetic radiation whose polarization is rotated by angle $\theta$ relative to the incoming optical pulse. For the opted configuration, the optical pulse propagates with an angle $\gamma$ relative to the $\mathrm{THz}$ propagation path, hence $\sin \gamma$ in Eq. (3) accounts for the projection of the $\mathrm{THz}$ magnetic field into the propagation direction of the optical pulse. As detailed in the Supplemental Material [32], under the influence of the $\mathrm{THz}$ magnetic field and after propagation of an effective distance $L_{\text {eff }}$ [40], the left and right circularly polarized components of the optical pulse undergo different phase shifts, thus the optical pulse acquires a polarization rotation

$$
\Delta \theta(t)=\frac{\pi L}{\lambda}\left(n_{-}-n_{+}\right)=R_{0} L_{\mathrm{eff}} \alpha^{2} B_{\mathrm{THz}}(t) \sin \gamma,
$$

where $R_{0}=\omega^{2} / 2 n_{0} e c$. It is interesting to note that, the rotation signal is related to the molecular property via its electric polarizability and to the optical pulse via its frequency, with no contribution of the optical electric-field amplitude $\mathbf{E}_{\text {opt }}$. As detailed in the Supplemental Material [32], in the weak interaction regime, $n_{L}$ and $n_{R}$ are obtained independent of $\mathbf{E}_{\mathrm{opt}}$, resembling the Hall angle in the classical Hall effect [41], in which it scales with the product of the magnetic field and the mobility of charge carriers.

Equation (4) indeed explains our main result, a response that scales linearly with the THz magnetic field $\mathbf{B}_{\mathrm{THz}}(t)$ and quadratically with the molecular polarizability. We show the soundness of the latter proportionality by plotting the $\Delta \theta$ signal amplitude vs the square of the average molecular polarizability of liquids. As shown in Fig. 5, there is a linear dependence of the square of the molecular polarizability and the measured rotation signals.

Finally, the Faraday rotation of highly polarizable molecular liquids such as $n$-hexane and 1 -hexanol exceeds that of TGG, more than twofold. Furthermore, TGG has a strong $\mathrm{THz}$ absorption and large refractive index, see Fig. 1(b), the latter of which causes the large velocity mismatch between $\mathrm{THz}$ and optical pulses, hence lowers the effective temporal resolution of the effect. In contrast, in nonpolar liquids, the $\mathrm{THz}$ absorption, and the velocity mismatch between the two pulses are negligible. Likewise, in long chain alcohols the $\mathrm{THz}$ absorption and the velocity mismatch decrease with increasing the alcohol chain length [45].

Competing nonlinear processes.-In the above description of the THz-magnetic-field induced Faraday rotation in liquids, we considered the optically induced polarization as the dominant contribution to the Hall current [see Eqs. (2) and (3)]. However, the THz electric field also contributes to the polarization build-up in liquids, thereby its impact appeals for a careful consideration. While, the first order polarization $P^{(1)}=\chi^{(1)} E_{\mathrm{THz}}$ has no impact on the optical probe polarization, higher order polarizations have [46]. In the employed $\mathrm{THz}$ pump-optical probe experiment, the dynamic THz Kerr effect (TKE) is the expected dominant third-order nonlinear effect from the bulk of liquids $[17,18]$, however, for the following reasons this effect is drastically reduced in our experiment. (i) The selected set of organic liquids possesses relatively small polarizability anisotropy $\Delta \alpha$. The TKE response scales with $\Delta \alpha^{2}$ in nonpolar liquids and with $\Delta \alpha$ in strongly polar systems; the small $\Delta \alpha$ of the selected liquids directly influences the amplitude of the TKE signal. (ii) In the optimum TKE configurations, the $\mathrm{THz}$ pump and the optical probe pulses propagate collinearly through the sample. The angle $\gamma$ in Fig. 1(a), reduces the amplitude of the TKE signal. (iii) The dynamic Kerr effect in liquids induces ellipticity in the optical probe pulse [18], thereby measuring the rotation instead of ellipticity further reduces the Kerr effect contribution on the $\Delta \theta(t)$ signal.

Although the second order nonlinear processes are forbidden in the bulk of materials with inversion symmetry, they are allowed on their interfaces, where the inversion symmetry is broken [47]. To examine the contribution of the surface nonlinear response $P^{(2)}=\chi^{(2)} E_{\mathrm{THz}} E_{\mathrm{optical}}$ on the rotation signals, which would also scale linearly with the $E_{\mathrm{THz}}$, we intersect the $\mathrm{THz}$ pump and the optical probe pulses in the depth of a liquid. As shown in Fig. S3 Supplemental Material [32], the mixing position of the two pulses has no impact on the observed rotation signal, dismissing the $P^{(2)}$ contribution on the rotation signals.

Does the $\mathrm{THz}$ magnetic field act on the electric-fieldinduced instantaneous polarization $\mathbf{P}_{e}(t)=\alpha \mathbf{E}_{\mathrm{THz}}(t)$ of the same pulse? If this effect occurs, its Hall current and accordingly its $\Delta \theta(t)$ signal would scale quadratically with the THz electric field, as $\mathbf{J}_{H}=\alpha \partial_{t} \mathbf{E}_{\mathrm{THz}}(t) \times \mathbf{B}_{\mathrm{THz}}(t)$ and $B_{\mathrm{THz}}(t)=E_{\mathrm{THz}}(t) / c$. However, the results in Fig. 3 show a linear relation of $\Delta \theta(t)$ and the THz field. Although, the measured $\Delta \theta(t)$ signals in Fig. 2 do not support observation 
of this effect, it might still exist but overlaid under the stronger Faraday rotation signal from $\mathbf{P}_{e}(t)=\alpha \mathbf{E}_{\text {opt }}(t)$.

In conclusion, THz-magnetic-field induced Faraday rotation in molecular liquids has been resolved on ultrafast timescales. The mechanism of the optical pulse polarization rotation is explained by the deflection of an optically induced instantaneous polarization in the presence of a $\mathrm{THz}$ magnetic field, in a manner analogous to the electric Hall effect in conducting materials. The observed effect scales linearly with the $\mathrm{THz}$ magnetic field and quadratically with the molecular polarizability. Highly polarizable, but lowly $\mathrm{THz}$ absorbing liquids such as long chain alcohols and alkanes show the largest effect. The ability of the transient molecular Hall effect to selectively resolve the electron dynamics in solutions, paves the way for the disentanglement of electronic vs nuclear dynamics in complex molecular systems. Moreover, the diverse physical and chemical properties of liquids may offer alternative solutions to the material incompatibility in traditional application of the Faraday effect in solid-state photonic devices [48].

We wish to thank Dr. Lukáš Nádvorník for fruitful discussions.

*Corresponding author. sajadi@fhi-berlin.mpg.de

[1] M. Inoue, M. Levy, and A. V. Baryshev, in Magnetophotonics (From Theory to Applications), Series in Materials Science (Springer, Heidelberg, 2013), p. 1782013.

[2] Y. Hibino, T. Koyama, S. Sumi, H. Awano, K. Miwa, S. Ono, M. Kohda, and D. Chiba, Appl. Phys. Express 10, 123201 (2017).

[3] Z. Jin, H. Ma, L. Wang, G. Ma, F. Guo, and J. Chen, Appl. Phys. Lett. 96, 201108 (2010).

[4] A. I. Lvovsky, B. C. Sanders, and W. Tittel, Nat. Photonics 3, 706 (2009).

[5] I. M. Savukov, S. K. Lee, and M. V. Romalis, Nature (London) 442, 1021 (2006).

[6] A. I. Musorin, M. I. Sharipova, T. V. Dolgova, M. Inoue, and A. A. Fedyanin, Phys. Rev. Applied 6, 024012 (2016).

[7] S. Schöche, P. Kühne, T. Hofmann, M. Schubert, D. Nilsson, A. Kakanakova-Georgieva, E. Janzén, and V. Darakchieva, Appl. Phys. Lett. 103, 212107 (2013).

[8] Y-F. Wu, D. Kapolnek, J. P. Ibbetson, P. Parikh, B. P. Keller, and U. K. Mishra, IEEE Trans. Electron Devices 48, 586 (2001).

[9] M. Suzuki, K. Fujii, T. Ohyama, H. Kobori, and N. J. Kotera, Phys. Soc. Jpn. 72, 3276 (2003).

[10] B. N. Szafranek, G. Fiori, D. Schall, D. Neumaier, and H. Kurz, Nano Lett. 12, 1324 (2012).

[11] S. Vandendriessche, S. Van Cleuvenbergen, P. Willot, G. Hennrich, M. Srebro, V. K. Valev, G. Koeckelberghs, K. Clays, J. Autschbach, and T. Verbiest, Chem. Mater. 25, 1139 (2013).
[12] R. R. Subkhangulov, R. V. Mikhaylovskiy, A. K. Zvezdin, V. V. Kruglyak, T. Rasing, and A. V. Kimel, Nat. Photonics 10, 111 (2016).

[13] R. R. Rau and M. E. Caspari, Phys. Rev. 100, 632 (1955).

[14] S. Kaka and S. E. Russek, Appl. Phys. Lett. 80, 2958 (2002).

[15] S. Kemmet, M. Mina, and R. J. Weber, J. Appl. Phys. 109, $07 \mathrm{E} 333$ (2011).

[16] J. Stake, T. Bryllert, T. A. Emadi, and J. Vukusic, Terahertz Frequency Detection and Identification of Materials and Objects (Springer, Dordrecht, 2007).

[17] M. C. Hoffmann, N. C. Brandt, H. Y. Hwang, K. L. Yeh, and K. A. Nelson, Appl. Phys. Lett. 95, 231105 (2009).

[18] M. Sajadi, M. Wolf, and T. Kampfrath, Nat. Commun. 8, 15796 (2017).

[19] H. A. Hafez, S. Kovalev, J-C. Deinert, Z. Mics, B. Green, N. Awari, M. Chen, S. Germanskiy, U. Lehnert, J. Teichert, Z. Wang, K-J. Tielrooij, Z. Liu, Z. Chen, A. Narita, K. Müllen, M. Bonn, M. Gensch, and D. Turchinovich, Nature (London) 561, 507 (2018).

[20] S. Fleischer, Y. Zhou, R. W. Field, and K. A. Nelson, Phys. Rev. Lett. 107, 163603 (2011).

[21] T. Kampfrath, A. Sell, G. Klatt, A. Pashkin, S. Mährlein, T. Dekorsy, M. Wolf, M. Fiebig, A. Leitenstorfer, and R. Huber, Nat. Photonics 5, 31 (2011).

[22] N. Kumar, A. C. Strikwerda, K. Fan, X. Zhang, R. D. Averitt, P. C. M. Planken, and A. J. L. Adam, Opt. Express 20, 11277 (2012).

[23] H. Yoshida, K. Tsubakimoto, Y. Fujimoto, K. Mika mi, H. Fujita, N. Miyanaga, H. Nozawa, H. Yagi, T. Yanagitani, Y. Nagata, and H. Kinoshita, Opt. Express 19, 15181 (2011).

[24] T. Fukasawa, T. Sato, J. Watanabe, Y. Hama, W. Kunz, and R. Buchner, Phys. Rev. Lett. 95, 197802 (2005).

[25] Y. Yomogida, Y. Sato, R. Nozaki, T. Mishina, and J. J. Nakahara, J. Mol. Liq. 154, 31 (2010).

[26] J. E. Pedersen and S. R. Keiding, IEEE J. Quantum Electron. 28, 2518 (1992).

[27] J. Hebling, K.-L. Yeh, M. C. Hoffmann, and K. A. Nelson, IEEE J. Sel. Top. Quantum Electron. 14, 345 (2008).

[28] H. Hirori, A. Doi, F. Blanchard, and K. Tanaka, Appl. Phys. Lett. 98, 091106 (2011).

[29] E. Molotokaite, V. Kumar, C. Manzoni, D. Polli, G. Cerullo, and M. J. Marangoni, Raman Spectrosc. 44, 1385 (2013).

[30] V. Kumar, M. Casella, E. Molotokaite, D. Gatti, P. Kukura, C. Manzoni, D. Polli, M. Marangoni, and G. Cerullo, Phys. Rev. A 86, 053810 (2012).

[31] M. Sajadi, M. Wolf, and T. Kampfrath, Opt. Express 23, 28985 (2015).

[32] See Supplemental Material at http://link.aps.org/ supplemental/10.1103/PhysRevLett.124.093201 for ellipticity and rotation signals of water, effect of the optical probe polarization on the rotation signal, surface nonspecificity of the rotation signal and the analytical model for the dynamic molecular Hall effect.

[33] T. Kampfrath, M. Wolf, and M. Sajadi, arXiv:1707. 07622v1.

[34] T. Kampfrath, R. K. Campen, M. Wolf, and M. Sajadi, J. Phys. Chem. Lett. 9, 1279 (2018). 
[35] J. A. Riordan, F. G. Sun, Z. G. Lu, and X. C. Zhang, Appl. Phys. Lett. 71, 1452 (1997).

[36] H. J. Bakker, G. C. Cho, H. Kurz, Q. Wu, and C. J. Zhang, J. Opt. Soc. Am. B 15, 1795 (1998).

[37] T. Hofmann, C. M. Herzinger, C. Krahmer, K. Streubel, and M. Schubert, Phys. Status Solidi A 205, 779 (2008).

[38] R. Bosque and J. J. Sales, J. Chem. Inf. Comput. Sci. 42, 1154 (2002).

[39] J.D. Jackson, Classical Electrodynamics 3rd ed. (John Wiley \& Sons, New York, 1999).

[40] While the height of liquids in the sample bucket is about a few millimeters, the intersection length of the $\mathrm{THz}$ and the optical pulses is $w_{\mathrm{THz}} \sin \gamma \approx 0.4 \mathrm{~mm}$, where $w_{\mathrm{THz}} \approx$ $0.6 \mathrm{~mm}$ is the $\mathrm{THz}$ beam waist at its focus. However, for liquids with high $\mathrm{THz}$ absorption the effective intersection length is given by $\delta_{p} \sin \gamma$, where $\delta_{p}$ is the THz penetration depth. As such, $L_{\text {eff }}$ is taken as the minimum of the latter two distances for each liquid.

[41] P. W. Anderson, Phys. Rev. Lett. 67, 2092 (1991).

[42] W. F. J. Murphy, Chem. Phys. 67, 5877 (1977).

[43] B. Ma, J-H. Lii, and N. L. Allinger, J. Comput. Chem. 21, 813 (2000).

[44] J. Richardi, P. H. Fries, and H. J. Krienke, J. Phys. Chem. B 102, 5196 (1998).

[45] S. Sarkar, D. Saha, S. Banerjee, A. Mukherjee, and P. Mandal, Chem. Phys. Lett. 678, 65 (2017).

[46] R. W. Boyd, Nonlinear Optics (Academic Press, San Diego, 1992).

[47] Y. R. Shen, Nature (London) 337, 519 (1989).

[48] L. Bi, J. Hu, P. Jiang, D. Hun Kim, G. F. Dionne, L. C. Kimerling, and C. A. Ross, Nat. Photonics 5, 758 (2011). 